\title{
MULTIVARIATE INTERPOLATION USING POLYHARMONIC SPLINES
}

\author{
KAREL SEGETH \\ Czech Academy of Sciences, Institute of Mathematics, Žitná 25, 11567 Praha 1, Czech Republic \\ correspondence: segeth@math.cas.cz
}

\begin{abstract}
Data measuring and further processing is the fundamental activity in all branches of science and technology. Data interpolation has been an important part of computational mathematics for a long time. In the paper, we are concerned with the interpolation by polyharmonic splines in an arbitrary dimension. We show the connection of this interpolation with the interpolation by radial basis functions and the smooth interpolation by generating functions, which provide means for minimizing the $\mathcal{L}^{2}$ norm of chosen derivatives of the interpolant. This can be useful in $2 \mathrm{D}$ and $3 \mathrm{D}$, e.g., in the construction of geographic information systems or computer aided geometric design. We prove the properties of the piecewise polyharmonic spline interpolant and present a simple 1D example to illustrate them.
\end{abstract}

KEYWORDS: Data interpolation, smooth interpolation, polyharmonic spline, Fourier transform.

\section{INTRODUCTION}

Measuring data of all different types and formats is the basic means of research in all branches of science and technology. It is a discrete process providing a finite number of numerical values over some domain. The first stage of data processing usually consists in its approximation, i.e., computing reliable data values at an arbitrary point in the domain of interest.

In the paper, we are concerned with the problem of data interpolation in an arbitrary dimension. In particular, we consider the interpolation with radial basis functions that is reasonable if we assume that the value at a point $x$ depends in some way on the Euclidean distance $r\left(x, X_{j}\right)$ between $x$ and the nodes $X_{j}$ where the values have been measured.

The background of the paper is the so-called smooth interpolation [1, 2] allowing for the minimization of some functionals applied to the interpolation formula. Choosing particular basis functions in the minimization space, we can get an interpolation formula whose principal part is a linear combination of polyharmonic splines of fixed order that are, at the same time, radial functions.

We construct such a radial basis, i.e. polyharmonic splines, and show its properties. Among other things, we prove that the interpolant is piecewise polyharmonic. We present a $1 \mathrm{D}$ example that shows the result of interpolation if different derivatives of the interpolant are minimized in the $\mathcal{L}^{2}$ norm. The example shows that the respective interpolations give expected results.

Interpolation of this nature is often employed in signal processing, construction of geographic information systems, or computer aided geometric design. Moreover, if the field measured is known to be polyharmonic, it is worth to interpolate it by a formula preserving the polyharmonicity.
Frequent citations of the author's paper [2] have been used to introduce the notation and basic properties of notions used. The conclusion of the present paper is more advanced, it shows that for interpolation it is possible to use polyharmonic functions of different orders $m$ that minimize different norms of the interpolant $u$, i.e. the $\mathcal{L}^{2}$ norm of the $(m+l)$ th derivative of $u$ for any $l$ positive.

We state the problem of data interpolation in Sec. 2 introduce radial functions in Sec. 3 and polyharmonic splines in Sec. 4. Further, we define the spaces $W_{L}$ where we are going to carry out the minimization and present a general form of the interpolation formula in Sec. 5. Moreover, we quote a theorem from [2] that states the existence and unicity of the solution of the interpolation problem. In Sec. 6, we choose exponential functions of a pure imaginary argument for the basis functions in $W_{L}$. For this choice of basis functions, we obtain a radial basis function interpolation formula, where the radial functions are polyharmonic functions, which can be seen in Sec. 7, present some properties (polyharmonicity) of such an interpolant in Sec. 8, and show a simple computional example in Sec. 9

\section{Problem of DAta interpolation}

Fundamental notation and basic statements are taken mostly from [2]. Consider a finite number $N$ of (complex, in general) measured (sampled) values $f_{1}, \ldots, f_{N} \in C$ obtained at $N$ given nodes $X_{1}, \ldots, X_{N} \in \Omega, X_{j}=\left(X_{j 1}, \ldots, X_{j n}\right)$, that are mutually distinct, where $n$ is a positive integer and $\Omega \in R^{n}$ is a cube. Usually, we need also the values corresponding to other points in $\Omega$ that are not known. Let $f_{j}=f\left(X_{j}\right)$ be measured values of a 
complex-valued function $f$ continuous in $\Omega$ and $z$ is an approximating function to be constructed.

Definition 1. The interpolating function (interpolant) $z$ is constructed to fulfill the interpolation conditions

$$
z\left(X_{j}\right)=f_{j}, \quad j=1, \ldots, N,
$$

cf. Definition 1 of [2]. Various additional conditions can be considered, e.g., minimization of some functionals applied to $z$.

The problem of data interpolation does not have a unique solution. The property (1) of the interpolant is clearly formulated by mathematical means but the behavior of the interpolating curve or the surface between nodes can differ case from case and cannot be formalized easily.

The problem of least squares data approximation is more general than the problem of data interpolation. No explicit interpolation conditions of the form (1) are to be satisfied, but the approximating function $z$ is constructed to minimize the least squares functional

$$
\sum_{j=1}^{N} w_{j}\left(z\left(X_{j}\right)-f_{j}\right)\left(z\left(X_{j}\right)-f_{j}\right)^{*}
$$

where $w_{j}, j=1, \ldots, N$, are positive weights and * denotes the complex conjugate, cf., e.g., [1]. Various additional conditions can be considered, for example, the minimization of some further functionals applied to $z$.

In some branches of science, the terminology may differ. The terms exact and inexact interpolation are also used if the interpolant or approximant satisfies the conditions (1) or not.

We are not concerned with the general data approximation in the paper.

\section{INTERPOLATION WITH RADIAL BASIS FUNCTIONS}

Let $x, y \in R^{n}$ and

$$
r(x, y)=\|x-y\|_{E}=\sqrt{\sum_{s=1}^{n}\left(x_{s}-y_{s}\right)^{2}}
$$

be the Euclidean norm of the vector $x-y \in R^{n}$. The dimension $n$ of the independent variable can be arbitrary.

Definition 2 (Radial function). We say that the function

$$
F(x, y)=\widehat{F}(r(x, y))
$$

depending only on $r$ from (2) is a radial function.

Radial functions are radially symmetric. They are often used as basis functions for interpolation as well as approximation. It is assumed that every item $f_{j}$ of the measured data at the node $X_{j}$ influences the result of interpolation or approximation at a point $x$ in the vicinity of $X_{j}$ proportionally, in some sense, to its distance $r\left(x, X_{j}\right)$ from $X_{j}$ if this vicinity can be considered "homogeneous".

The vector $\alpha=\left(\alpha_{1}, \ldots, \alpha_{n}\right)$, where $\alpha_{s}, s=$ $1, \ldots, n$, are integers, is called a multiindex. Denote the length of a multiindex $\alpha$ by

$$
|\alpha|=\sum_{s=1}^{n}\left|\alpha_{s}\right|
$$

where $\left|\alpha_{s}\right|$ means the absolute value of the component $\alpha_{s}$. We say that $\alpha$ is a nonnegative multiindex if $\alpha_{s} \geq 0$ holds for all $s=1, \ldots, n$.

Choose a nonnegative integer $L$ and consider the interpolant

$$
z(x)=\sum_{j=1}^{N} \lambda_{j} F\left(x, X_{j}\right)+\sum_{|\alpha| \leq L-1} a_{\alpha} \varphi_{\alpha}(x),
$$

where $\alpha$ is a nonnegative multiindex, $F(x, y)=$ $\widehat{F}(r(x, y))$ is a radial basis function (e.g. a proper polyharmonic spline, see Sec. 4), $\varphi_{\alpha}$ are all the monomials of the form

$$
\varphi_{\alpha}(x)=x_{1}^{\alpha_{1}} \ldots x_{n}^{\alpha_{n}}
$$

of degree $|\alpha| \leq L-1$ called trend functions, and $\lambda_{j}, j=1, \ldots, N$, and $a_{\alpha},|\alpha| \leq L-1$, are coefficients to be found; the second sum in the formula (4) is empty if $L=0$ (cf. [2]).

The interpolation with radial basis functions is widely used in computational practice. For practical reasons, the basis functions are usually taken only from a very small set of functions, e.g., $\sqrt{r^{2}+s^{2}}$, $1 / \sqrt{r^{2}+s^{2}}, \exp \left(-s r^{2}\right)$, or $r^{2} \ln (r / s)$, where $s>0$ is a constant.

\section{Polyharmonic splines}

Definition 3 (Polyharmonic spline). Let $r(x, y)$ be the Euclidean norm (2) of the vector $x-y \in R^{n}$. The functions

$$
\begin{aligned}
r^{q}, & q=1,3, \ldots, \\
r^{q} \ln r, & q=2,4, \ldots,
\end{aligned}
$$

are called polyharmonic splines.

The equation

$$
\Delta^{m} u\left(x_{1}, \ldots, x_{n}\right)=0,
$$

where $\Delta=\partial^{2} / \partial x_{1}^{2}+\cdots+\partial^{2} / \partial x_{n}^{2}$ is the Laplace operator, is called the polyharmonic equation of order $m$, cf. 22. Apparently, all the derivatives in the equation (8) are of order $2 m$. Polyharmonic splines solve the respective polyharmonic equation. The next theorem presents the exact statement. 
Theorem 1. Fix the vector $y \in R^{n}$. Then the polyharmonic spline $r^{q}$ (or $r^{q} \ln r$ ) solves the polyharmonic equation in variable $x$ of order $m=\frac{1}{2}(q+n)$ in $R_{y}^{n}=R^{n} \backslash\{x=y\}$ for $n$ odd (or for $n$ even).

Proof. It is easy to prove the statement by direct computation.

Note that the term spline is used here also for a nonpolynomial function. Another (weak) definition of the polyharmonic spline can be given with the help of the Dirac function.

Apparently, $r(x, y)$ is a real radial basis function. All polyharmonic splines (6), (7) also possess the same property. For a practical use in approximation, the polyharmonic splines are combined with lower order polynomial terms (trends) to form an interpolation or approximation formula as in (4).

\section{Smooth interpolation}

Let us briefly present some properties of polyharmonic splines in the smooth approximation or variational spline theory. These properties show the place of splines in the context of radial basis function interpolation. Alternatively, the splines can be derived with the help of the algebraic spline theory, cf. an example of the $1 \mathrm{D}$ cubic spline in 3 .

We employ the usual Lebesgue space $\mathcal{L}^{2}(\Omega)$ of generalized complex-valued functions with the norm

$$
\|g\|_{\mathcal{L}^{2}}^{2}=\int_{\Omega}|g(x)|^{2} \mathrm{~d} x .
$$

We follow 1 and 4, and formulate and solve the problem of smooth interpolation [2]. Choose a set $\left\{B_{\alpha}\right\}$ of nonnegative numbers, where $\alpha$ is a nonnegative multiindex. Let $L$ be the smallest nonnegative integer such that $B_{\alpha}>0$ for at least one $\alpha,|\alpha|=L$, while $B_{\alpha}=0$ for all $\alpha,|\alpha|<L$.

Recall that the nodes $X_{1}, \ldots, X_{N} \in \Omega$ are supposed to be mutually distinct. Let $\widetilde{\mathcal{W}}$ be a linear vector space of complex valued functions $g$ continuous together with all their partial derivatives of all orders in $\Omega$. For $g, h \in \widetilde{\mathcal{W}}$ we put

$$
\begin{aligned}
& (g, h)_{L} \\
& =\sum_{L \leq|\alpha|} B_{\alpha} \int_{\Omega} \frac{\partial^{|\alpha|} g(x)}{\partial x_{1}^{\alpha_{1}} \ldots \partial x_{n}^{\alpha_{n}}}\left(\frac{\partial^{|\alpha|} h(x)}{\partial x_{1}^{\alpha_{1}} \ldots \partial x_{n}^{\alpha_{n}}}\right)^{*} \mathrm{~d} x
\end{aligned}
$$

and similarly

$$
|g|_{L}^{2}=\sum_{L \leq|\alpha|} B_{\alpha} \int_{\Omega}\left|\frac{\partial^{|\alpha|} g(x)}{\partial x_{1}^{\alpha_{1}} \ldots \partial x_{n}^{\alpha_{n}}}\right|^{2} \mathrm{~d} x
$$

if the values of $|g|_{L}$ and $|h|_{L}$ exist and are finite.

If $L=0$ (i.e. $B_{\alpha}>0$ for $|\alpha|=0$ ), consider functions $g, h \in \widetilde{\mathcal{W}}$ such that the values of $|g|_{0}$ and $|h|_{0}$ exist and are finite. Then $(g, h)_{0}$ has the properties of inner product and the expression $\|g\|_{0}=|g|_{0}$ is norm in a normed space $W_{0}=\widetilde{\mathcal{W}}$.

Let $L>0$. Consider again functions $g, h \in \widetilde{\mathcal{W}}$ such that the values of $|g|_{L}$ and $|h|_{L}$ exist and are finite. Let $P_{L-1} \subset \widetilde{\mathcal{W}}$ be the subspace whose basis $\left\{\varphi_{\alpha}\right\}$, where $\alpha$ is a nonnegative multiindex, $|\alpha| \leq L-1$, consists of all the trend functions (5) of degree $L-1$, at most. Then, for a nonnegative multiindex $\beta$,

$$
\begin{aligned}
& \left(\varphi_{\alpha}, \varphi_{\beta}\right)_{L}=0 \text { and }\left|\varphi_{\alpha}\right|_{L}=0 \\
& \quad \text { for }|\alpha| \leq L-1 \text { and }|\beta| \leq L-1 .
\end{aligned}
$$

Using (9) and (10), we construct the quotient space $\widetilde{\mathcal{W}} / P_{L-1}$ whose zero class is the subspace $P_{L-1}$. Finally, considering $(\cdot, \cdot)_{L}$ and $|\cdot|_{L}$ in every equivalence class, we see that they represent the inner product and norm $\|g\|_{L}$ in the normed space $W_{L}=\widehat{\mathcal{W}} / P_{L-1}$.

$W_{L}$ is the normed space where we minimize functionals and measure the smoothness of the interpolation as prescribed by the choice of $\left\{B_{\alpha}\right\}$. We complete the space $W_{L}$ in the norm $\|\cdot\|_{L}$ and denote the completed space again $W_{L}$. For an arbitrary $L \geq 0$, choose a basis system of functions $\left\{g_{\varkappa}\right\} \subset W_{L}$ that is complete and orthogonal (in the inner product in $W_{L}$ ), i.e., if $\varkappa=\left(\varkappa_{1}, \ldots, \varkappa_{n}\right)$ and $\mu=\left(\mu_{1}, \ldots, \mu_{n}\right)$ are nonnegative multiindices then

$$
\left(g_{\varkappa}, g_{\mu}\right)_{L}=0 \quad \text { for } \quad \varkappa \neq \mu .
$$

If $L>0$ then, moreover,

$$
\left(\varphi_{\alpha}, g_{\varkappa}\right)_{L}=0 \text { for a nonnegative multiindex } \alpha \text {, }
$$

$$
|\alpha| \leq L-1 \text {. }
$$

The set $\left\{\varphi_{\alpha}\right\}$ of trend functions is empty for $L=0$.

Definition 4 (Smooth interpolation). The problem of smooth interpolation [1] consists in finding the complex coefficients $A_{\varkappa}$ and $a_{\alpha}$ of the interpolant

$$
z(x)=\sum_{\varkappa} A_{\varkappa} g_{\varkappa}(x)+\sum_{|\alpha| \leq L-1} a_{\alpha} \varphi_{\alpha}(x)
$$

with nonnegative multiindices $\varkappa$ and $\alpha$ such that

$$
z\left(X_{j}\right)=f_{j}, \quad j=1, \ldots, N,
$$

and

the quantity $\|z\|_{L}^{2}$ attains its minimum on $W_{L}$.

The second sum in the interpolant (14) is empty for $L=0$. According to 10 , the quantity $\|z\|_{L}^{2}$ is the weighted sum of the squares of $\mathcal{L}^{2}$ norms of the derivatives of $z$ of all orders $|\alpha|$ with weights $B_{\alpha}$. Putting $B_{\alpha}>0$ for some set of multiindices $\alpha$, we can specify the partial derivatives of $z$ whose $\mathcal{L}^{2}$ norms are to be minimized, i.e., the smoothness of the interpolant $z$. For example, if $n=1$ we put $B_{k}=0$, except for $B_{2}=1$ (i.e. $L=2$ ), and minimize the $\mathcal{L}^{2}$ 
norm of the second derivative of $z$, which corresponds to minimizing its curvature.

Apparently,

$$
\|z\|_{L}^{2}=\sum_{\varkappa} A_{\varkappa} A_{\varkappa}^{*}\left\|g_{\varkappa}\right\|_{L}^{2}
$$

due to $11,12,13)$, and $(14)$.

Remark 1. With a fixed $n$, it is easy to employ the multinomial theorem to find out (see [2]) that there are

$$
\Pi(n,|\alpha|)=\left(\begin{array}{c}
|\alpha|+n-1 \\
n-1
\end{array}\right)
$$

mutually different nonnegative multiindices $\alpha$ of $n$ components with $|\alpha|$ fixed. The same is the number of the trend functions $\varphi_{\alpha}$ with $|\alpha|$ fixed and

$$
T(n, L)=\sum_{|\alpha| \leq L-1}\left(\begin{array}{c}
|\alpha|+n-1 \\
n-1
\end{array}\right)=\left(\begin{array}{c}
L-1+n \\
n
\end{array}\right)
$$

is the total number of the trend functions $\varphi_{\alpha},|\alpha| \leq$ $L-1$.

To remove the inconvenient infinite sum from (14), we introduce the generating function [1].

Definition 5 (Generating function). Let the basis system of functions $\left\{g_{\varkappa}\right\} \subset W_{L}$, where $\varkappa$ is a nonnegative multiindex, be complete and orthogonal in $W_{L}$. If the series

$$
R(x, y)=\sum_{\varkappa} \frac{g_{\varkappa}(x) g_{\varkappa}^{*}(y)}{\left\|g_{\varkappa}\right\|_{L}^{2}}
$$

converges for all $x, y \in \Omega$ and is continuous in $\Omega$ we call the fuction $R(x, y)$ the generating function.

If $L>0$, introduce an $N \times T(n, L)$ matrix $\Phi$ with entries

$$
\Phi_{j \alpha}=\varphi_{\alpha}\left(X_{j}\right), \quad j=1, \ldots, N,|\alpha| \leq L-1 .
$$

The matrix $\Phi$ is, in general, rectangular.

We state in following Theorem 2 that a finite linear combination of the values of the generating function $R(x, y)$ at nodes is used for the practical interpolation instead of the infinite linear combination of the values of the basis functions in (14).

Theorem 2. Let $X_{i} \neq X_{j}$ for all $i \neq j$. Assume that the series (17) converges for all $x, y \in \Omega$ and the generating function $R(x, y)$ is continuous in $\Omega$. Moreover, let rank $\Phi=T(n, L)$. Then the problem (14), (15), and (16) of smooth interpolation has the unique solution

$$
z(x)=\sum_{j=1}^{N} \lambda_{j} R\left(x, X_{j}\right)+\sum_{|\alpha| \leq L-1} a_{\alpha} \varphi_{\alpha}(x),
$$

where the complex, in general, coefficients $\lambda_{j}, j=$ $1, \ldots, N$, and $a_{\alpha},|\alpha| \leq L-1$, are the unique solution of the linear algebraic system

$$
\begin{aligned}
& \sum_{j=1}^{N} \lambda_{j} R\left(X_{i}, X_{j}\right)+\sum_{|\alpha| \leq L-1} a_{\alpha} \varphi_{\alpha}\left(X_{i}\right)=f_{i}, \\
& i=1, \ldots, N,=0, \\
& \sum_{j=1}^{N} \lambda_{j} \varphi_{\alpha}^{*}\left(X_{j}\right) \\
&|\alpha| \leq L-1 .
\end{aligned}
$$

Proof. The proof is given in [2].

Note that we have to solve the linear algebraic system 19$), 20)$ for $N+T(n, L)$ unknowns. The number of unknowns (and equations) depends on $n$ only through $T(n, L)$, the number of trend functions.

The smooth interpolant $z$ given by $(14)$ can now be rewritten for the generating function $R(x, y)$ in the form (18).

\section{A PERIODIC BASIS FUNCTION SYSTEM OF $W_{L}$}

Let the continuous function $f(x)=f\left(x_{1}, \ldots, x_{n}\right)$, to be interpolated, be $2 \pi$-periodic in each independent variable $x_{s}, s=1, \ldots, n$. Periodic functions with other periods in the individual variables can be formally transformed to the period $2 \pi$. Let us consider $f$ in the cube $\widetilde{\Omega}=[0,2 \pi]^{n}$. Write

$$
x \cdot y=x_{1} y_{1}+\cdots+x_{n} y_{n}
$$

for the $R^{n}$ inner product of vectors $x$ and $y$.

We choose exponential functions of a pure imaginary argument for the periodic basis system $\left\{g_{\rho}\right\}$ in $W_{L}$, where $g_{\rho}(x)=\exp (-\mathrm{i} \rho \cdot x)$. We have to change the notation properly with respect to the fact that the integer components of the multiindex $\rho$ are also negative. The definition (3) of the length $|\rho|$ of the multiindex $\rho$ remains without change. In the definition (17) of the generating function $R(x, y)$, we sum over all multiindices $\rho$ (not only over those with nonnegative components). The following theorem shows important properties of the system $\left\{g_{\rho}\right\}$.

Theorem 3. Let there be an integer $U, U \geq L$, such that $B_{\alpha}=0$ for all $|\alpha|>U$ in $W_{L}$. The system of periodic exponential functions of pure imaginary argument

$$
g_{\rho}(x)=\exp (-\mathrm{i} \rho \cdot x), x \in \widetilde{\Omega},
$$

$\rho$ being a multiindex with integer components $\rho_{s}=$ $0, \pm 1, \pm 2, \ldots, s=1, \ldots, n$, is complete and orthogonal in $W_{L}$.

Proof. The proof is given in [2]. 
Remark 2. Note that on the assumption of Theorem 3 that there is an integer $U$ of required properties, $B_{\alpha}>0$ can occur only for $L \leq|\alpha| \leq U$. We will keep this assumption in the rest of the paper.

We further follow [2]. For the basis system (21), notice that $\rho$ is not nonnegative and the generating function

$$
\begin{aligned}
R(x, y) & =\sum_{\rho} \frac{g_{\rho}(x) g_{\rho}^{*}(y)}{\left\|g_{\rho}\right\|_{L}^{2}} \\
& =\sum_{\rho} \frac{\exp (-\mathrm{i} \rho \cdot(x-y))}{\left\|g_{\rho}\right\|_{L}^{2}}
\end{aligned}
$$

is the $n$-dimensional Fourier series in $\mathcal{L}^{2}(\widetilde{\Omega})$ with the coefficients $\left\|g_{\rho}\right\|_{L}^{-2}$, where

$$
\left\|g_{\rho}\right\|_{L}^{2}=(2 \pi)^{n} \sum_{|\alpha|=L}^{U} B_{\alpha} \rho_{1}^{2 \alpha_{1}} \ldots \rho_{n}^{2 \alpha_{n}}
$$

according to 10 .

Let now the complex-valued function $f$, to be interpolated, be nonperiodic in $R^{n}$. Redefine the generating function

$$
\begin{aligned}
R(x, y) & =\int_{R^{n}} \frac{\exp (-\mathrm{i} \rho \cdot(x-y))}{\left\|g_{\rho}\right\|_{L}^{2}} \mathrm{~d} \rho \\
& =\mathcal{F}\left(\frac{1}{\left\|g_{\rho}\right\|_{L}^{2}}\right)
\end{aligned}
$$

as the $n$-dimensional Fourier transform $\mathcal{F}$ of the function $\left\|g_{\rho}\right\|_{L}^{-2}$ of $n$ continuous variables $\rho_{1}, \rho_{2}, \ldots, \rho_{n}$ if the integral exists 4. Employing the transition from the Fourier series 22 with the coefficients $\left\|g_{\rho}\right\|_{L}^{-2}$ to the Fourier transform 23 of the function $\left\|g_{\rho}\right\|_{L}^{-2}$ of continuous variable $\rho \in R^{n}$ (cf., e.g., [5]), we have transformed the basis functions, enriched their spectrum, and released the requirement of periodicity of $f$. Moreover, if the integral (23) does not exist in the usual sense, in many instances, we can calculate $R(x, y)$ as the Fourier transform $\mathcal{F}$ of the generalized function $\left\|g_{\rho}\right\|_{L}^{-2}$ of $\rho$.

The generating function $R(x, y)$ given by 23$)$ depends on $x$ and $y$ only through the distance $r(x, y)$.

\section{Polyharmonic SPline INTERPOLATION}

In the notation introduced above, we continue in deriving the polyharmonic spline interpolation according to [2]. Put

$$
K(\alpha)=\frac{|\alpha| !}{\alpha_{1} ! \ldots \alpha_{n} !}
$$

for a nonnegative multiindex $\alpha$. Recall that $n$ is the dimension of the problem, fix $L>0$, and put $B_{\alpha}=0$ for all $\alpha,|\alpha| \neq L$, and $B_{\alpha}=K(\alpha)$ for $|\alpha|=L$. Then

$$
\left\|g_{\rho}\right\|_{L}^{2}=(2 \pi)^{n}\left(\sum_{s=1}^{n} \rho_{s}^{2}\right)^{L}
$$

according to the multinomial theorem.

In tables (e.g. [6]), we easily find

$$
\begin{aligned}
& R(x, y)=\mathcal{F}\left(\left(\sum_{s=1}^{n} \rho_{s}^{2}\right)^{-L}\right) \\
& =\left\{\begin{array}{l}
C_{1} r^{2 L-n} \text { for } n \text { odd } \\
C_{21} r^{2 L-n} \ln r+C_{22} r^{2 L-n} \text { for } n \text { even, }
\end{array}\right.
\end{aligned}
$$

where $r=r(x, y)$ is given by (2) and $C_{1}, C_{21}$, and $C_{22}$ are quantities depending only on $n$ and $L$. Then the generating function $R(x, y)$, for $2 L-n>0$, is a radial basis function.

Note that the function 25 has the form of the polyharmonic function (6) only if the dimension $n$ is odd and it is the sum of the polyharmonic function (7) and $C_{22} r^{2 L-n}$ if $n$ is even. Using Lemmas 2 and 3 of 2, we remove the term $C_{22} r^{2 L-n}$ from the formula 25 for the generating function in the case of $n$ being even. We obtain

$$
\begin{aligned}
R(x, y) & =r^{2 L-n} \text { for } n \text { odd } \\
& =r^{2 L-n} \ln r \text { for } n \text { even. }
\end{aligned}
$$

For $2 L-n>0$, the generating function $R(x, y)$ is the polyharmonic spline (6) or (7), i.e., a radial basis function.

\section{SOME PROPERTIES OF THE POLYHARMONIC INTERPOLANT}

Consider now the interpolant $z$ given by 18 where the generating function $R(x, y)$ is the polyharmonic spline for $n$ odd (26) or for $n$ even (27), $n$ fixed. Its properties are characterized by the following theorem and lemma.

Theorem 4. Choose $L$ such that $2 L-n>0$. Let the interpolant $z(x)$ be given by $(18)$. Then it solves the polyharmonic equation (8) of order $m=L$ in the set $R_{X}^{n}=R^{n} \backslash \bigcup_{j=1}^{N}\left\{x=X_{j}\right\}$.

Proof. According to Theorem 1, the generating function $R\left(x, X_{j}\right)=r^{2 L-n}\left(x, X_{j}\right)$ for $n$ odd or $R\left(x, X_{j}\right)=$ $r^{2 L-n}\left(x, X_{j}\right) \ln r\left(x, X_{j}\right)$ for $n$ even, it is the solution of the polyharmonic equation of order $m=$ $\frac{1}{2}(2 L-n+n)=L$ in $R_{X_{j}}^{n}, j=1, \ldots, N$. Moreover, the trend functions $\varphi_{\alpha}(x)$ given by $(5)$ are monomials of a degree at most $L-1$. They satisfy the polyharmonic equation of order $m=L$ in $R^{n}$ as the operator $\Delta^{L}$ is a linear combination of the derivatives of order $2 L$ and, according to the multinomial theorem, each of these derivatives includes a derivative of order $L$ with respect to some particular variable $x_{s}$. The coefficients $\lambda_{j}$ and $a_{\alpha}$ are complex constants. Therefore, the interpolant (18) satisfies the polyharmonic equation (8) in $R_{X}^{n}$. 


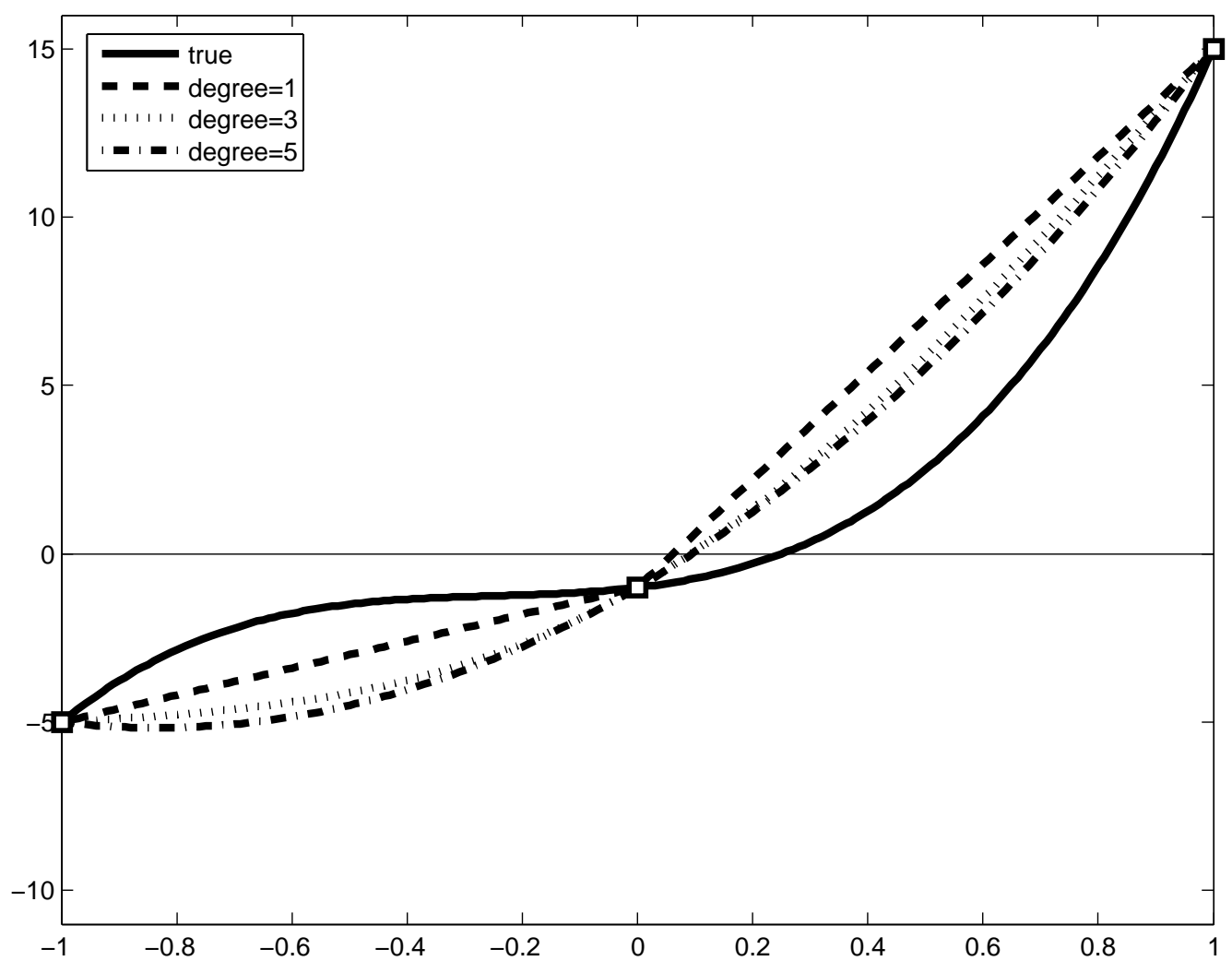

Figure 1. $N=5$. The horizontal axis: independent variable, the vertical axis: the true function 29 (solid line); the interpolant with $B_{1}=1$ (dashed line, piecewise linear), $B_{2}=1$ (dotted line, cubic spline), and $B_{3}=1$ (dash-dot line, quintic spline). The scales on the $x$ - and $y$-axis are different.

We have just proven that the interpolant 18 with the generating function (26) or 27) is polyharmonic in $R_{X}^{n}$. Moreover, in the example we will use its another trivial property stated in the following lemma.

Lemma 1. Let the function $u$ of the variable $x=$ $\left(x_{1}, x_{2}, \ldots, x_{n}\right)$ satisfy the polyharmonic equation of order $m$ in the set $\Psi \subset R^{n}$. Then it satisfies the polyharmonic equation of order $m+l$ for any positive $l$ in the same set.

\section{EXAMPLE}

In Fig. 1, we show results of a simple computation: the polyharmonic spline interpolation for $n=1$, i.e. the modification

$$
z(x)=\sum_{j=1}^{N} \lambda_{j} R\left(x, X_{j}\right)+\sum_{k=0}^{L-1} a_{k} \varphi_{k}(x)
$$

of the formula $(18)$ with $R(x, y)$ given by 26$)$. Note that $\alpha=k$ is now a simple index. We consider three cases: the minimization of the $\mathcal{L}^{2}$ norm of the 1 st, or 2nd, or 3rd derivative of the interpolant. We interpolate the third degree polynomial

$$
f(x)=8 x^{3}+6 x^{2}+2 x-1
$$

on $\Omega=[-1,1]$ (solid line in Fig. 1) with $N=3$, i.e. using the nodes $X_{1}=-1, X_{2}=0$, and $X_{3}=1$. We employ the formula (24) for $K(\alpha)$ in case $n=1$, i.e.,
$K(L)=1$, and put $B_{k}=0$ for all $k, k \neq L$, and $B_{L}=1$.

If we put $L=1, B_{1}=1$, and $B_{k}=0$ otherwise to minimize the $\mathcal{L}^{2}$ norm of the 1 st derivative of the interpolant according to 16 then the generating function $R(x, y)=r^{2 L-n}=r$ (a piecewise linear function given by (2) ) and the trend function is a polynomial of degree $L-1=0$, i.e. a constant. The interpolant (28) solves the equation (8), which is now harmonic $(m=L=1)$, according to Theorem 4 , everywhere in $R^{1}$ except for the points $x=X_{j}, j=$ 1,2,3 (cf. Sec. 8). The constant trend function satisfies the equation (8) everywhere in $R^{1}$.

From the form of the interpolation formula 28 we see that the interpolant $z(x)$ (dashed line in Fig. 1) does not satisfy the equation (8) at the three nodes $X_{1}, X_{2}, X_{3}$. This is not important at the first and last node where the value prescribed can be understood as a boundary condition. We can thus claim that the interpolant (28) satisfies the harmonic equation (8) on $(-1,1) \backslash\{0\}$. Moreover, according to Lemma 1 the interpolant $z(x)$ given by (28) also satisfies the polyharmonic equation (8) of any order $m>1$ in the same set as the equation of order 1 , i.e. on $(-1,1) \backslash\{0\}$.

If we further put $L=2, B_{2}=1$, and $B_{k}=0$ otherwise to minimize the $\mathcal{L}^{2}$ norm of the 2 nd derivative of the interpolant, then $R(x, y)=r^{2 L-n}=r^{3}$ (the well-known cubic spline), the trend functions are a constant and linear function. The interpolant (28) 
(dotted line in Fig. 1) solves the biharmonic equation (8) as $m=L=2$ according to Theorem 4 everywhere in $R^{1}$ except for the points $x=X_{j}, j=1,2,3$. The constant and linear trend functions satisfy the equation (8) everywhere in $R^{1}$. As in the previous case, we see that the interpolant 28 satisfies the biharmonic equation $(8)$ on $(-1,1) \backslash\{0\}$. Again, according to Lemma 1, the interpolant $z(x)$ also satisfies the polyharmonic equation (8) of any order $m>2$ in the same set.

If we finally put $L=3, B_{3}=1$, and $B_{k}=0$ otherwise to minimize the $\mathcal{L}^{2}$ norm of the 3 rd derivative of the interpolant, then $R(x, y)=r^{2 L-n}=r^{5}$ (quintic spline), the trend functions are a constant, linear function, and a quadratic function. The interpolant (28) (dash-dot line in Fig. 1) solves the triharmonic equation (8) as $m=L=3$ by Theorem 4 everywhere in $R^{1}$ except for the points $x=X_{j}, j=1,2,3$. All the trend functions satisfy the equation (8) everywhere in $R^{1}$. As in the previous case, we see that the interpolant (28) satisfies the triharmonic equation (8) on $(-1,1) \backslash\{0\}$. According to Lemma 1 the interpolant $z(x)$ satisfies the polyharmonic equation (8) of any order $m>3$ in the same set.

The results agree with general expectations. The interpolation conditions (1) are satisfied. For $n=1$, the formula (2) gives $r(x, y)=|x-y|$, i.e. the absolute value of the difference $x-y$. Naturally, minimizing the $\mathcal{L}^{2}$ norm of the first derivative of the interpolant $\left(B_{1}=1\right)$ gives a broken line. Minimizing the same norm of the second derivative of the interpolant (its curvature) using $B_{2}=1$ leads to a cubic spline. If we put $B_{3}=1$, we minimize the $\mathcal{L}^{2}$ norm of the third derivative of the interpolant by a quintic spline.

Apparently, it is not possible to draw principal conclusions from a single $1 \mathrm{D}$ example. $2 \mathrm{D}$ and $3 \mathrm{D}$ cases are more interesting and can be applied to many problems of practice.

\section{Conclusion}

Using the general theory of smooth interpolation we constructed a radial basis interpolant as a linear combination of the values of a polyharmonic spline of fixed order and a linear combination of the trend functions. The construction shows how to choose the functional applied to the formula in order to minimize particular derivatives of the interpolant, i.e., to get the smoothness of these derivatives. Moreover, the interpolant is proven to be piecewise polyharmonic, which can be considered advantageous in some cases. Note that the problem considered is $n$-dimensional and that the number of equations of the linear algebraic system to be solved is the number $N$ of nodes of the measurement plus the number of trends (that depends on the dimension).

\section{ACKNOWLEDGEMENTS}

The author was supported by RVO 67985840 and by Czech Science Foundation grant 18-09628S.

\section{REFERENCES}

[1] A. Talmi, G. Gilat. Method for smooth approximation of data. J Comput Phys 23:93-123, 1977. DOI:10.1016/0021-9991(77)90115-2

[2] K. Segeth. Polyharmonic splines generated by multivariate smooth interpolation. Comput Math Appl 78:3067-3076, 2019. DOI:10.1016/j.camwa.2019.04.018

[3] K. Segeth. Some splines produced by smooth interpolation. Appl Math Comput 319:387-394, 2018. DOI:10.1016/j.amc.2017.04.022

[4] L. Mitáš, H. Mitášová. General variational approach to the interpolation problem. Comput Math Appl 16:983-992, 1988. DOI:10.1016/0898-1221(88)90255-6.

[5] K. Segeth. A periodic basis system of the smooth approximation space. Appl Math Comput 267:436-444, 2015. DOI:10.1016/j.amc.2015.01.120.

[6] S. G. Kreĭn (ed.). Functional analysis (Russian). 1st edition. Nauka, Moskva, 1964 